\title{
AN EXAMINATION OF 3D, BROADBAND ACOUSTIC PROPAGATION PHYSICS IN A LITTORAL OCEAN ENVIRONMENT - AN EXTENSION TO AN ONR PRIMER FIELD STUDY IN THE MID-ATLANTIC BIGHT
}

\author{
Kevin B. Smith \\ Department of Physics, Code PH/Sk \\ Naval Postgraduate School \\ Monterey, CA 93943 \\ phone: (408) 656-2084 fax: (408) 656-2834 email: kevin@usw.nps.navy.mil \\ Award \#: N0001497WR30001
}

\section{LONG-TERM GOAL}

The overall goal of this project is to improve our understanding of acoustic propagation in complicated littoral zones near shelf breaks. Of particular interest to me are the effects of the continental slope, shelf break front, and internal soliton waves on the propagation of broadband signals and the significance of 3-D azimuthal (out-of-plane) coupling.

\section{SCIENTIFIC OBJECTIVES}

The objective of this work is to quantify the significance of 3-D azimuthal coupling effects on the pulse propagation due to the sloping bathymetry and the shelf break front. The variability (both spatially and temporally) of plane wave arrivals will also be quantified and related to fluctuations caused by tidal cycles and interactions with internal wave soliton packets. The ability to determine which frequencies propagate most readily and which environmental parameters dominate over particular frequency bands will also be investigated.

\section{APPROACH}

Data taken from the NW VLA of the PRIMER III Mid-Atlantic Bight experiment (Pickart et al, 1996) will be the focus of the processing in this work. This array measured acoustic data on 16 elements spanning the water column for most of the experiment while the three southern tomography sources $(400+/-50 \mathrm{~Hz})$ were transmitting PRN sequences. A P-3 flight took place on one day which deployed broadband explosive SUS sources in the oparea. During this portion of the experiment, only the upper half of the NW VLA recorded data. In addition to the acoustic data, the Woods Hole SeaSoar group was recording water column data in part to provide sound speed information. Bathymetric data for this region was also available for modeling purposes. 


\section{Report Documentation Page}

Form Approved

OMB No. 0704-0188

Public reporting burden for the collection of information is estimated to average 1 hour per response, including the time for reviewing instructions, searching existing data sources, gathering and maintaining the data needed, and completing and reviewing the collection of information. Send comments regarding this burden estimate or any other aspect of this collection of information,

including suggestions for reducing this burden, to Washington Headquarters Services, Directorate for Information Operations and Reports, 1215 Jefferson Davis Highway, Suite 1204, Arlington

VA 22202-4302. Respondents should be aware that notwithstanding any other provision of law, no person shall be subject to a penalty for failing to comply with a collection of information if it

does not display a currently valid OMB control number.

1. REPORT DATE

30 SEP 1997

2. REPORT TYPE

3. DATES COVERED

00-00-1997 to 00-00-1997

4. TITLE AND SUBTITLE

An Examination of 3D, Broadband Acoustic Propagation Physics in a Littoral Ocean Environment - an Extension to an ONR Primer Field Study in the Mid-Atlantic Bight

5a. CONTRACT NUMBER

5b. GRANT NUMBER

5c. PROGRAM ELEMENT NUMBER

6. AUTHOR(S)

5d. PROJECT NUMBER

5e. TASK NUMBER

5f. WORK UNIT NUMBER

7. PERFORMING ORGANIZATION NAME(S) AND ADDRESS(ES)

Naval Postgraduate School,Department of Physics,Monterey,CA,93943

8. PERFORMING ORGANIZATION

REPORT NUMBER

9. SPONSORING/MONITORING AGENCY NAME(S) AND ADDRESS(ES)

10. SPONSOR/MONITOR'S ACRONYM(S)

11. SPONSOR/MONITOR'S REPORT

$\operatorname{NUMBER}(\mathrm{S})$

12. DISTRIBUTION/AVAILABILITY STATEMENT

Approved for public release; distribution unlimited

13. SUPPLEMENTARY NOTES

14. ABSTRACT

15. SUBJECT TERMS

16. SECURITY CLASSIFICATION OF:

\begin{tabular}{c|c|c}
$\begin{array}{c}\text { a. REPORT } \\
\text { unclassified }\end{array}$ & $\begin{array}{c}\text { b. ABSTRACT } \\
\text { unclassified }\end{array}$ & $\begin{array}{c}\text { c. THIS PAGE } \\
\text { unclassified }\end{array}$
\end{tabular}

17. LIMITATION OF ABSTRACT

Same as

Report (SAR)
18. NUMBER 19a. NAME OF

OF PAGES

7 RESPONSIBLE PERSON 


\section{WORK COMPLETED}

The cruise took place from 19 July to 9 August and most instrumentation worked as expected. Acoustic data from the VLA's has been processed at WHOI, URI, and NPS, and is available for study.

In order to study the acoustic variability in this region, the NW VLA data recording the SW $400 \mathrm{~Hz}$ tomography source has been matched filtered and coherently averaged over 5 minutes to provide arrival time information every 15 minutes (each tomo source "sang" for 5 minutes and was quiet for 10). This data has been plane wave beamformed every 15 minutes over the course of several hours. It was also beamformed every hour (every 4th data set) over the course of several days. Trends in the data are being analyzed, variability due to tides and mesoscale advection is being investigated, and ping-to-ping correlations are being computed for various arrival angles.

An investigation into optimal propagation frequency bands is on-going. This work has been delayed primarily because we have not been able to form a good match between observation and model predictions. It is currently assumed that this is due to the lack of good bottom parameter estimation. Methods of inverting for geo-acoustic properties are being considered.

The determination of the influence of 3-D azimuthal coupling has been purely based on modeling up to this point. A 3-D version of the Monterey-Miami Parabolic Equation (MMPE) model (Smith, 1996) has been upgraded and is being tested against potential benchmark scenarios. Previous results (Smith, 1997) are suspect at this time, and a reinvestigation of the influence due to bathymetry alone (iso-speed water) has been completed. The inclusion of 3-D variable water column properties is currently being analyzed.

\section{RESULTS}

The data displayed in Figure 1 show the signal variability over several hours of the central (horizontal) beam arrival. In Figure 2, this same beam data is displayed for several days. The results of the 15 minute ping-to-ping correlation show very little coherence over this time scale for the center, or any, beam angles. The data compiled over several days does exhibit a $\sim 12$ hour tidal cycle as well as an overall increase in arrival times due to a cold shelf break water intrusion. It is unclear at this time whether this longer time trend is due simply to a decrease in sound speed (due to decreasing temperature) or whether propagation path lengths have been increased due to the different refractive characteristics of the colder medium. 
An empirical estimate of a SUS source spectrum was used with the measured pressure levels to extract an estimate of the broadband transmission loss to the NW VLA. An example of this is shown in Figure 3. While the environmental mechanisms responsible for the general shape of this curve are still being investigated, there appears to be an overall energy peak below about $1 \mathrm{kHz}$ with secondary peaks at $\sim 50 \mathrm{~Hz}$ and possibly at $\sim 400$ Hz. Current model predictions produce widely differing results depending on the properties of the sediment. In order to more fully understand the frequency dependent nature of the observed signal levels, more definitive estimations of the geo-acoustic parameters are needed.

Predictions of the arrival time structures at the NW VLA from the SE tomography source using the 2-D and 3-D versions of MMPE are displayed in Figures 4 and 5, respectively. These results show only the significance of the sloping bathymetry since the water column sound speed was assumed to be iso-speed. A comparison of these two data sets show no measurable difference, suggesting that azimuthal coupling due to shelf-break bathymetry is insignificant over these ranges.

\section{IMPACT/APPLICATION}

The apparent insignificance of 3-D effects found here should justify the use of simpler (and faster) 2-D models in similar environments, particularly if these results hold when 3-D sound speed variations are included. Future results from the study of environmental impacts on optimal propagation frequencies may lead to improved sonar system designs and/or processing techniques in littoral regions. The extremely small ping-to-ping correlation times of beam data in shallow water environments may suggest limits on the usefulness of current/future coherent inter-ping processing methods.

\section{TRANSITIONS}

None at this time.

\section{RELATED PROJECTS}

1 - Ching-Sang Chiu (NPS) has been considering similar variability issues in the context of modes.

2 - Jim Miller (URI) has been performing geo-acoustic inversion calculations using a propagation model with genetic algorithm techniques.

\section{REFERENCES}


Pickart, R. S., Gawarkiewicz, G. G., Lynch, J. F., Chiu, C.-S., Smith, K. B., Miller, J. H., "Endeavor 286 Cruise Summary: PRIMER III," Cruise Report, 1996.

Smith, K.B., "Modeling the effects of azimuthal coupling on acoustic propagation in the presence of 3-dimensional, rough ocean interfaces using the parabolic approximation," Special Issue of Theoretical and Computational Acoustics, D. Lee, Y-H Pao, M.H. Shultz, and Y.-C. Teng (eds), World Scientific Publishing Co., 115-131, 1996. (Invited paper)

Smith, K.B., "Three-dimensional effects on broadband pulse propagation near shelf breaks," Proceedings of International Conference on Shallow Water Acoustics, Beijing, China, April 21- 25, 1997.

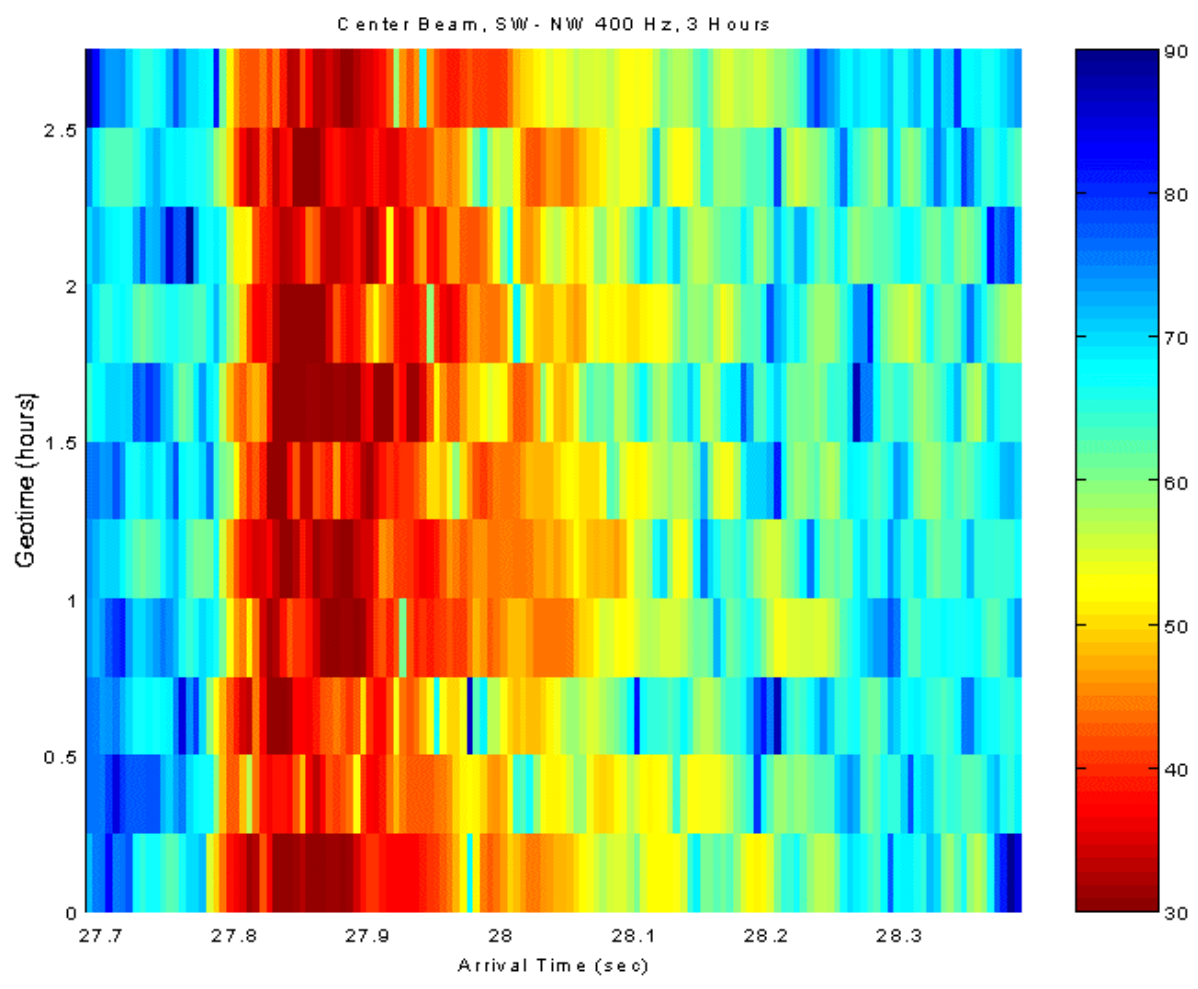




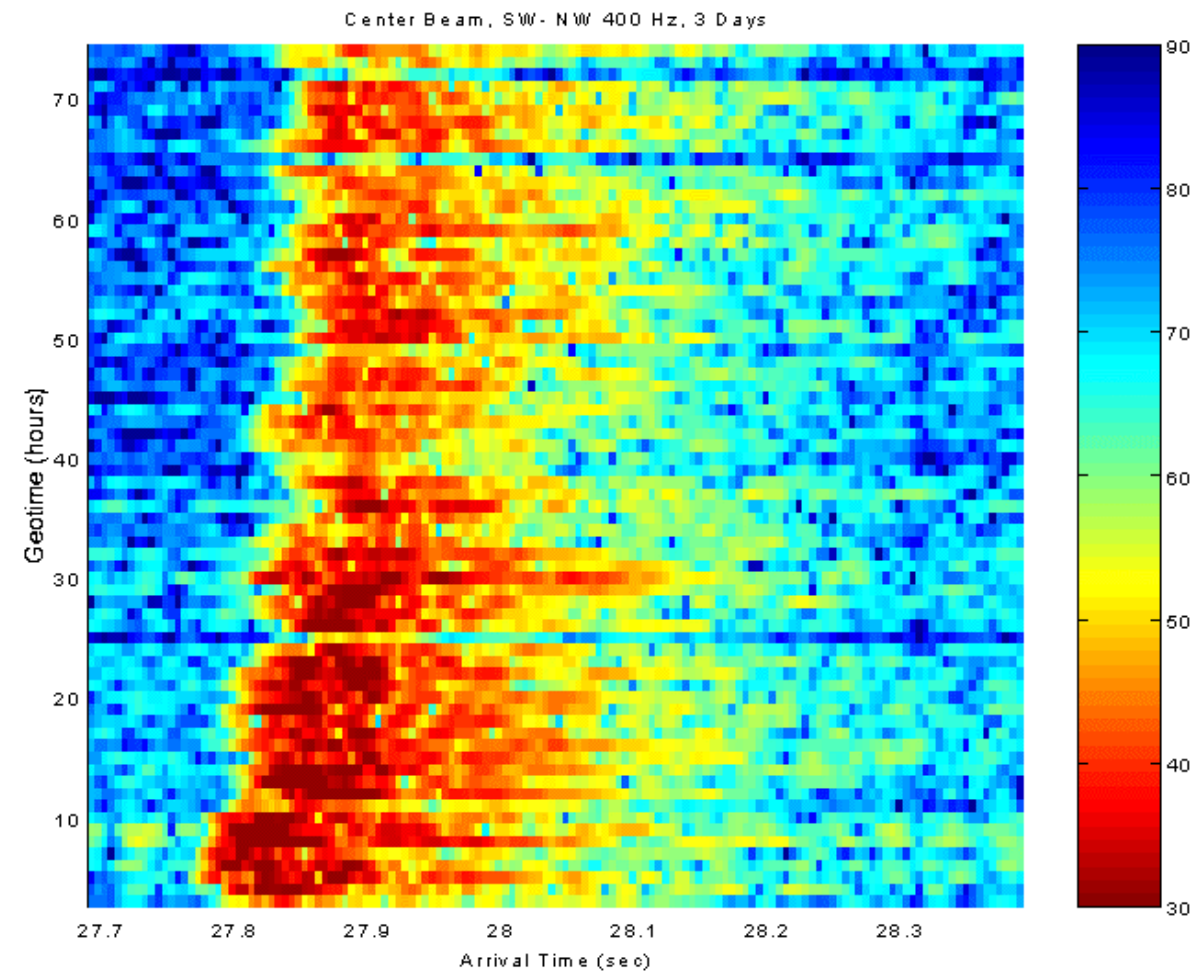




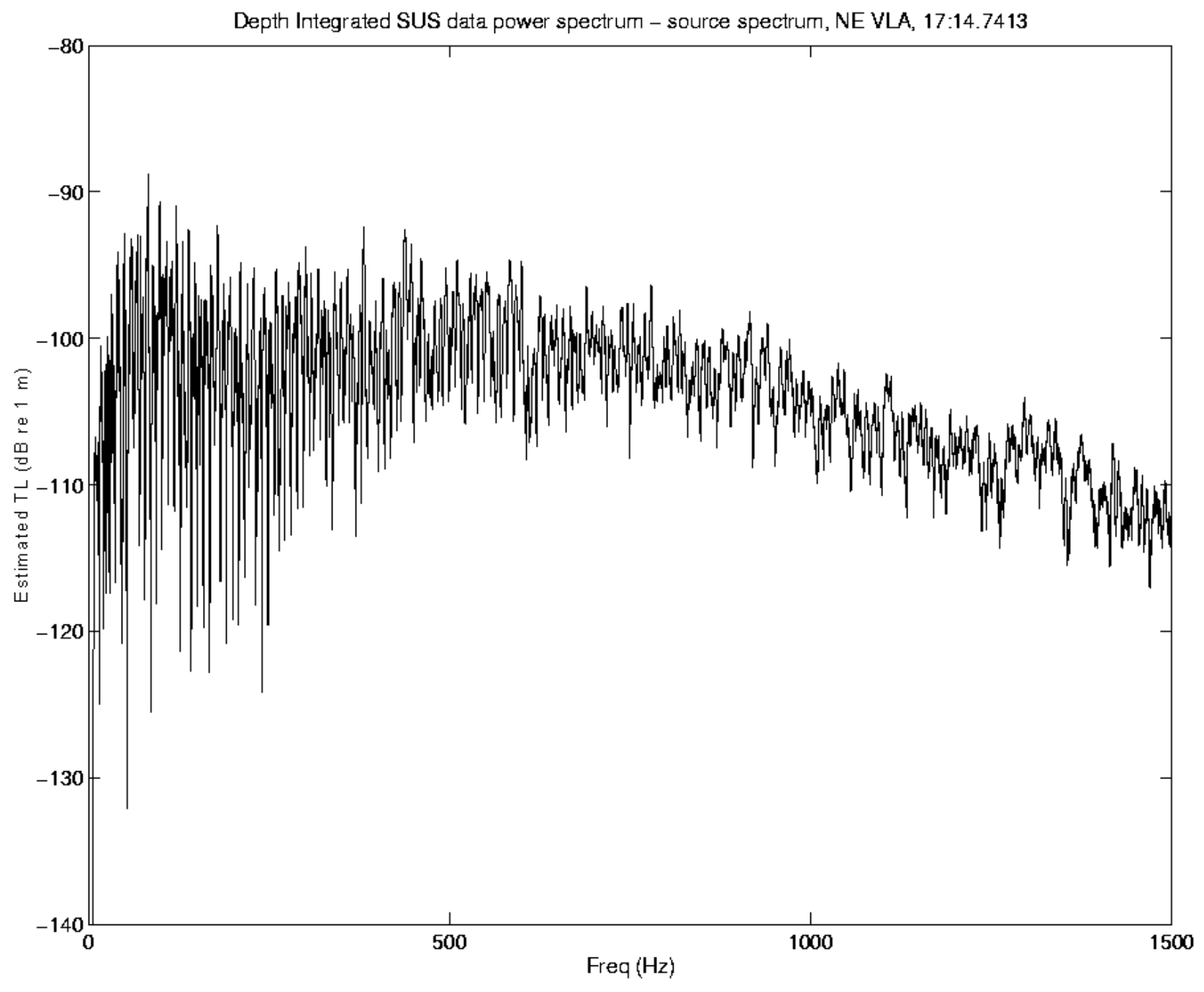



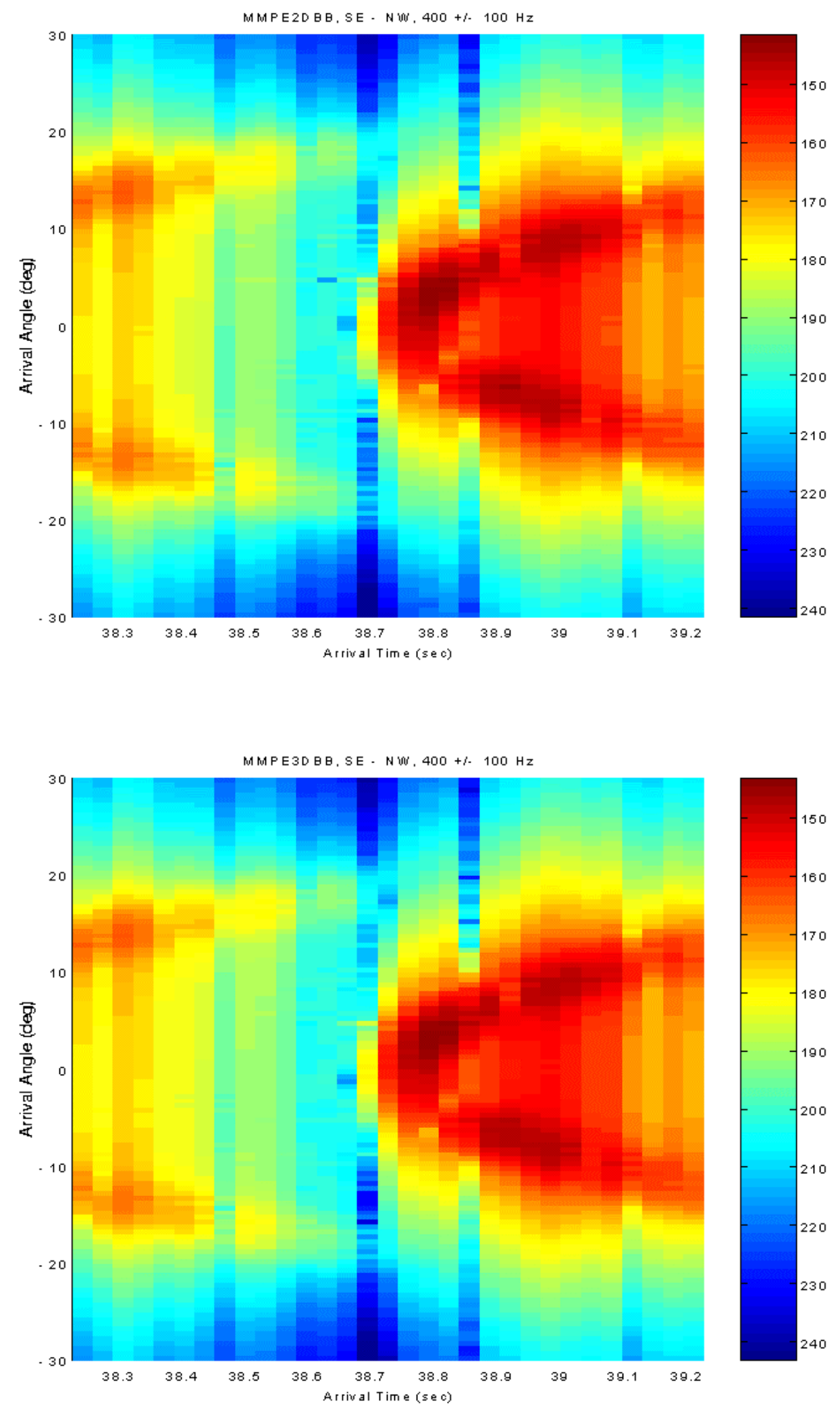